\title{
Laparoscopic restaging of ovarian granulosa cells tumor: three cases report
}

\author{
Eugenio Volpi • Annamaria Ferrero • Elisa Cassina • \\ Francesca Pastorino
}

Received: 31 December 2007 / Accepted: 11 April 2008/Published online: 27 May 2008

(C) Springer-Verlag 2008

\begin{abstract}
Granulosa cell tumor (GCT) of the ovary is an uncommon neoplasm, often diagnosed at an early stage. Surgery is necessary for definitive diagnosis and staging. Complete staging is recommended as for epithelial ovarian cancer and a restaging procedure is sometime required. The staging procedure should include evaluation of para-aortic and pelvic lymph nodes. We report three cases of laparoscopic restaging of GCT. The patients were treated in our Institution from August 2005 to January 2007. They were diagnosed an ovarian cyst and underwent laparoscopic adnexectomy. Histology showed a GCT (one case of juvenile type in a 38-year-old woman). All the patients were submitted to laparoscopic restaging with pelvic and para-aortic lymphadenectomy. Mean operation time was $195 \mathrm{~min}$. A mean number of 32 lymph nodes were removed. No major intraoperative or postoperative complication occurred. Patients were discharged on the second or third postoperative day. All the patients are well and without disease. Laparoscopic restaging of GCT is feasible and allows para-aortic and pelvic lymphadenectomy. Patients restaged by laparoscopy benefit of a less invasive treatment.
\end{abstract}

E. Volpi $\cdot$ A. Ferrero $\cdot$ E. Cassina $\cdot$ F. Pastorino

Department of Gynaecologic Oncology, University of Turin, c/o ASO Ordine Mauriziano,

Largo Turati,

62-10128 Turin, Italy

E. Volpi $\cdot$ A. Ferrero $\cdot$ E. Cassina $\cdot$ F. Pastorino

Institute for Cancer Research and Treatment,

Candiolo, Turin, Italy

Present address:

A. Ferrero $(\bowtie)$

Via G. Carducci, 20-10044,

Pianezza, Turin, Italy

e-mail: a.ferrero@katamail.com
Keywords Granulosa cell tumor - Laparoscopic surgery . Ovarian cancer $\cdot$ Lymphadenectomy

\section{Introduction}

Granulosa cell tumor (GCT) is a rare stromal cell neoplasm that represents less than $5 \%$ of all ovarian malignancies, but $70 \%$ of malignant sex-cord stromal tumor. GCT is divided into adult and juvenile types based on different clinical and histological features. Juvenile GCT represents only $5 \%$ of cases. GCT usually occurs in the perimenopausal or early menopausal period, but the juvenile type is most common in prepubertal girls or women younger than 30 years. GCT typically presents at an early stage. Patients may have symptoms of hyper-estrogenism associated with a pelvic mass, but diagnosis is rare before surgery $[1,2]$.

Surgery is the mainstay of initial management for patients with a suspected GCT. It is necessary to establish a definitive tissue diagnosis, perform staging or restaging in early stages, and debulk gross disease in advanced stages. A complete staging as for epithelial ovarian cancer is recommended [1]. Diagnosis is frequently performed at definitive histology and a restaging procedure may be required [3]. A restaging is most important especially when the disease is apparently limited to the ovary because of the prognostic role of stage [4].

Usually, staging and restaging are performed by laparotomy, while the role of laparoscopy is not yet clear [2]. In the last two decades, laparoscopic surgery has greatly changed the approach to ovarian masses, and most of them are treated laparoscopically. Nevertheless, laparoscopic staging for early ovarian malignancies remains controversial [5].

The staging procedure should include evaluation of paraaortic and pelvic lymph-nodes [1]. At the beginning of the 
nineties, laparoscopic pelvic and para-aortic lymphadenectomies were first described by different authors. Evaluating the reliability and safety of laparoscopic lymph-node dissection in a large-scale study on 1,000 gynecologic cancer patients, Querleu et al. concluded that laparoscopic surgery may be considered as the gold standard of assessment of the status of regional lymph-nodes in gynecological malignancies [6]. On the other hand, in clinical practice laparoscopy is not the most common surgical access for ovarian cancer staging.

We report 3 cases of GCT in which the first procedure and the restaging, including para-aortic and pelvic lymphadenectomy, were performed laparoscopically.

\section{Case 1 report}

A 38-year-old woman, PARA 3003, came to the emergency ward with abdominal pain and nausea. At palpation, pain in right pelvic zone was found. Ultrasound (US) showed a mass of fluid content $(15 \mathrm{~cm})$.

According to the diagnosis of right ovarian torsion, the patient underwent right laparoscopic adnexectomy. Histology showed a GCT with cystic appearance and juvenile growth (Fig. 1). No tumor stage was applicable because of fragmentation of the cyst, but spillage was avoided during extraction.

After 1 month, she was submitted to complete laparoscopic restaging, including left salpingo-oophorectomy, laparoscopically assisted vaginal hysterectomy, peritoneal washing and biopsies, and pelvic and para-aortic lymphadenectomy. The total duration of the operation was $215 \mathrm{~min}$. Thirty-two nodes were removed: 27 para-aortic and five pelvic. Histological examination did not find sign of residual disease: no evidence of peritoneal spread was found; all lymph-nodes were normal or hyperplastic, without metastasis.

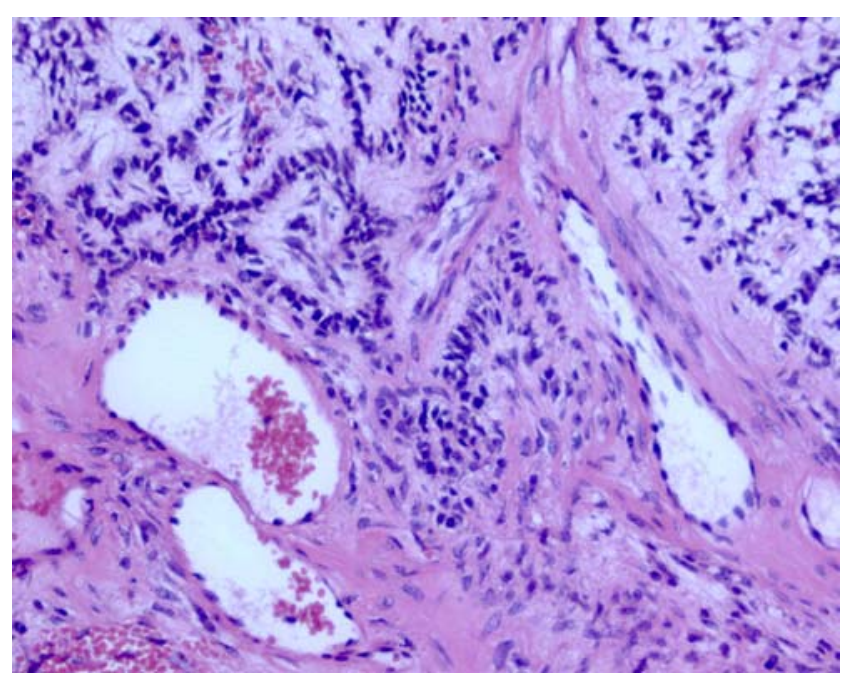

Fig. 1 Histologic pattern of a juvenile granulosa cell tumor
The patient was discharged on the second postoperative day. No major intraoperative or postoperative complication occurred. No adjuvant therapy was administered. During the last follow-up control at 2 years the patient resulted without disease.

\section{Case 2 report}

A 51-year-old woman, PARA 3013, during an US examination for colecystic calculosis presented two left ovarian cystic masses $(5$ and $3 \mathrm{~cm}$ ), with thick septa but without flow at US-color doppler. About 1 month later, she underwent a trans-vaginal US that diagnosed a hemorrhagic luteal cyst with the persistence of the ovarian mass. The serum levels of Ca 125 (29 mU/l), CEA (1,4 mU/l), and AFP (4,6 mU/l) were in the normal range.

The patient was proposed laparoscopic treatment. Left adnexectomy was performed and the histological examination showed an adult GCT (Fig. 2).

After 1 month, she was submitted to laparoscopic restaging. Laparoscopically assisted vaginal hysterectomy, right salpingo-oophorectomy, peritoneal washing and biopsies were performed. Furthermore, 34 nodes ( 11 pelvic and 23 para-aortic) were removed. The total duration of the intervention was $195 \mathrm{~min}$. The duration of para-aortic lymph node dissection was $75 \mathrm{~min}$. No histological evidence of peritoneal spread was found, and examination of all lymph nodes does not reveal any sign of disease, but peritoneal washing was positive. Definitive FIGO stage was Ic.

The patient was discharged on the third postoperative day. No major intraoperative or postoperative complication occurred.

After 5 months, the patient was submitted to a laparoscopic second look because cytology was positive at restaging. The duration of operation was $25 \mathrm{~min}$. The

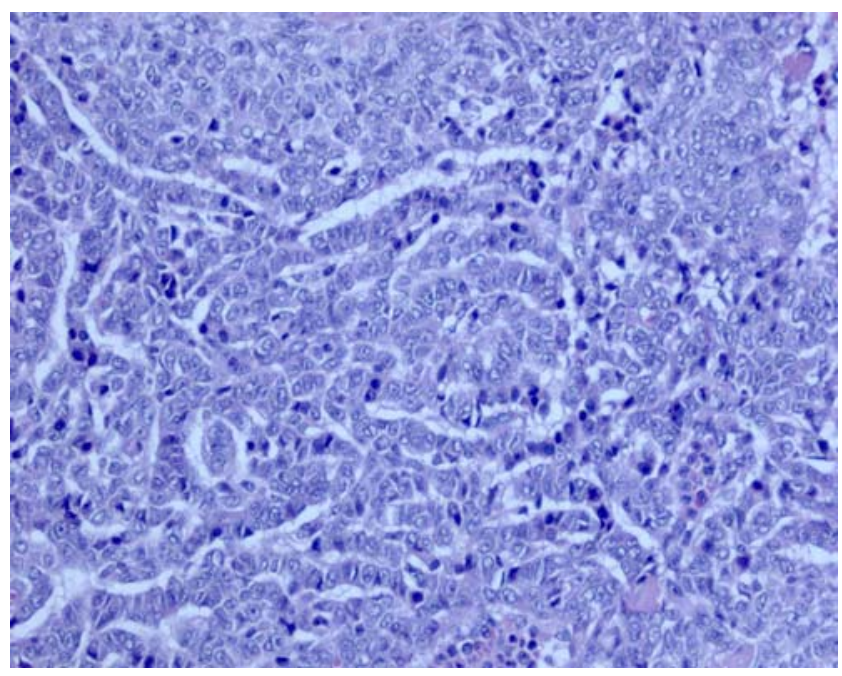

Fig. 2 Trabecular pattern and granulosa cells with characteristic "coffee-bean" nuclei in an adult granulosa cell tumor 
patient was discharged on the first postoperative day. During the operation, no residual neoplasm was found, and no adjuvant therapy was given. Further 18 months later, the patient is healthy.

\section{Case 3 report}

A 47-year-old woman, PARA 2012, was admitted in our department with diagnosis of adult GCT, occasional medical report on voluminous left ovarian cistoma removed by laparoscopy.

She was submitted to laparoscopic restaging with peritoneal cytology and biopsies, pelvic and para-aortic lymphadenectomy. After the evidence of a ventrofixation of the uterus, hysterectomy and right salpingo-oophorectomy were performed with a Pfamnestiel incision. Twenty-five nodes (nine pelvic and 16 para-aortic) were removed. The total duration of the operation was $180 \mathrm{~min}$. The duration of lymph node dissection was $90 \mathrm{~min}$. Restaging was negative for residual disease.

The patient was discharged on the third postoperative day. No major intraoperative or postoperative complication occurred. At the 6 months control the patient was well.

\section{Laparoscopic para-aortic lymph-node dissection technique}

Laparoscopy instruments are conventional. Placement of the trocars is in the usual diamond position. Hemostasis is accomplished by means of bipolar coagulation exclusively. Dissection is started from the right common iliac vessels, proximal to the right ureter, after placing the patient in steep Trendelemburg position. The peritoneum is opened up to the Treitz angle. At this step, the camera is rotated so that the aorta is seen horizontally. We start from the left side identifying first the aortic bifurcation (Fig. 3). To go further with the dissection, a fifth ancillary trocar is placed below the ninth coast. Lymphadenectomy is then started from the left renal vein coming caudally first to the inferior

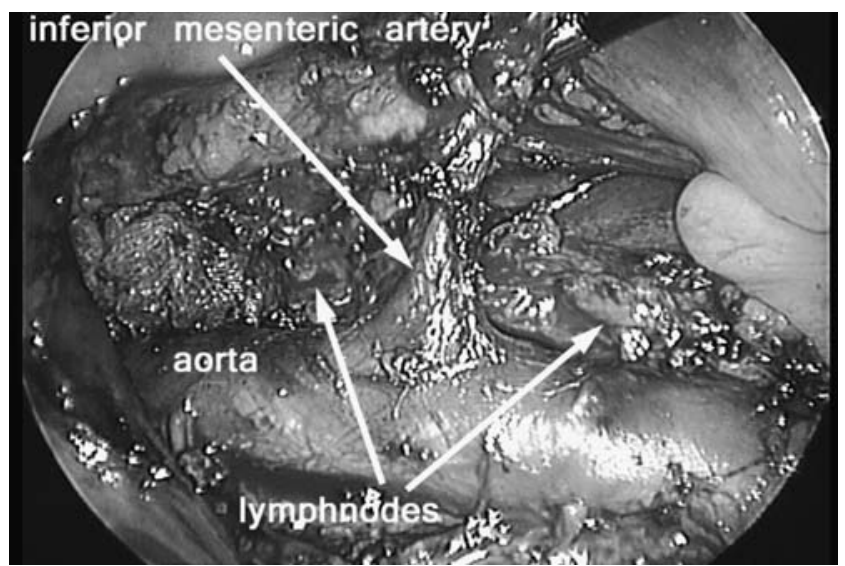

Fig. 3 Identification of the aorta and the inferior mesenteric artery

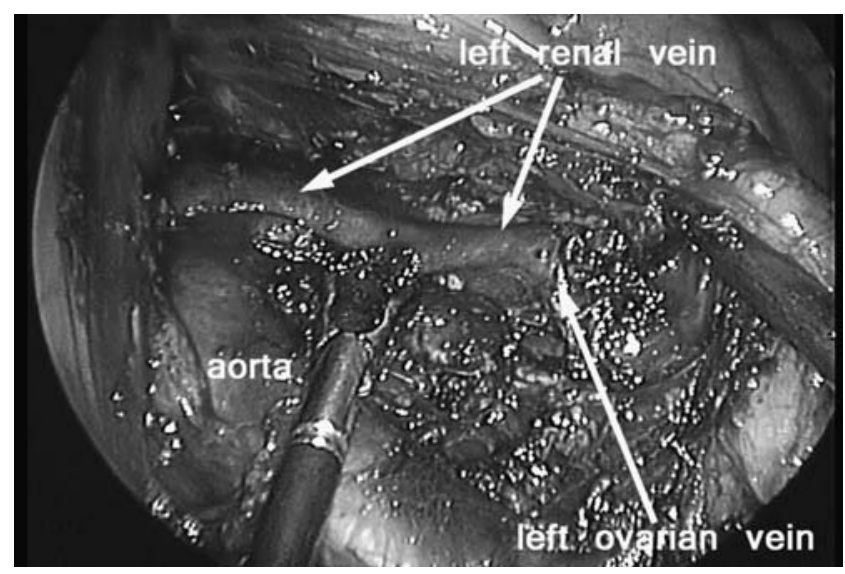

Fig. 4 Dissection to the left renal vein and identification of the left ovarian vein

mesenteric artery and then from it to the common iliac paying attention to the lumbar arteries (Fig. 4). Then, we start the right part of the procedure over the vena cava. The last lymph nodes dissected are the right common iliac where perforating vessels are described.

\section{Discussion}

The primary treatment of ovarian GCT is surgery and the surgical principles are generally identical to those used in the management of epithelial ovarian cancer. Staging procedures are the same and a laparotomy should be performed trough a midline incision $[1,2]$. In fact it is still the general agreement that a patient with ovarian cancer should have laparotomy to ensure optimal staging and treatment. Laparoscopy is a releasable tool in early ovarian carcinoma. Tozzi et al. [5] reported a series of 24 patients with FIGO stage IA-B ovarian cancer submitted to primary treatment or restaging by laparoscopy. The staging was performed according to the FIGO guidelines, and the conclusion was that laparoscopic management of early ovarian cancer is safe and effective, and survival outcome seems acceptable. Moreover, in a review [7], the authors asserted that the use of laparoscopy is safe when oncologic guidelines are respected. Ghezzi et al. [8] compared the results of laparoscopic staging (15 patients) of apparent early ovarian cancer with those obtained with comprehensive surgical staging via laparotomy (19 patients). They concluded that laparoscopic surgical staging of epithelial ovarian cancer is as safe and adequate as the laparotomic one.

In 1994, Querleu [9] reported the first case of laparoscopic surgical therapy and staging of early malignant GCT of the ovary. The patient was submitted to laparoscopic staging including pelvic and para-aortic dissection; she was discharged on the third day and was doing well 22 months postoperatively. 
Despite the role of minimally invasive surgery in children with ovarian pathologies remains controversial, diagnostic laparoscopy has been accepted as a standard to identify the origin of a tumor, the extent of disease, and the tumor's respectability. In 2005, Till and Schmidt [10] reported the first evidence that laparoscopic resection of a juvenile GCT (stage FIGO 1a) can achieve long-term oncological success. A 6-year-old girl underwent laparoscopy, and there was no evidence of tumor recurrence after more than 3 years.

Stage of disease has been shown to be the most important prognostic factor in most studies [4]. It has been reported that patients with stage I disease who underwent adequate staging procedure have a very low risk of recurrence [11]. If disease was not staged at first surgery, a restaging intervention should be performed.

In our three cases, laparoscopy was safe and effective. No spillage occurred during the first diagnostic approach, and a complete staging was possible during the restaging procedure. An advantage of laparoscopy is that all the peritoneal surfaces and lymph nodes can be carefully inspected and examined by the laparoscope magnification.

Our patient with juvenile GCT was older than usual for this histotype. Nevertheless, she is doing well after 2 years. Recently, Zhang et al. [4] reported an analysis of 376 women with sex-cord stromal tumors (339 with granulosa cell tumors), that represents the largest series in the literature. They concluded that age $\leq 50$ and early-stage disease are significant prognostic factors for improved survival. All our patients were less than 50 years old and had a stage 1 disease. These positive prognostic factors could influence the results of the laparoscopic approach.

There are no data to support any kind of postoperative adjuvant treatment for patients with stage I GCTs, given the indolent nature of these neoplasms and the overall good prognosis [11]. One of the patients was classified as FIGO 1c after restaging. Since only cytology was positive, we discussed about chemotherapy versus a second-look procedure at 6 months. After the second look, the patient was negative to inspection, biopsies, and cytology. Now, after about 1 year and half, the patient is without disease. Correct staging is necessary to decide further therapies.

GCT can recur years after initial diagnosis and prolonged follow-up is required [11]. All our patients will be followed in our institution. Despite the follow-up period is short till now, all our patients are well. We cannot draw any conclusions from the oncologic point of view, but we think that the laparoscopic approach does not interfere with prognosis, especially for early-stage disease.

In women of childbearing age and with disease limited to one ovary, a fertility-sparing surgery can be a reasonable approach [11]. In a cohort study on 376 women, Zhang et al. [4] reported that a conservative surgical treatment appears to be a safe alternative for young patients with early stage disease. In postmenopausal women, bilateral salpingo-oophorectomy with total hysterectomy is recommended, since GCT is often associated with endometrial hyperplasia or even endometrial cancer [3, 11]. All our patients were in the perimenopausal period, and hysterectomy was always performed.

While lymphadectomy is recommended for early-stage ovarian cancer, it is yet unclear for sex cord stromal tumors. As for epithelial ovarian cancer, the staging procedure includes evaluation of para-aortic and pelvic lymph-nodes [1]. Some authors sustain that in the majority of cases, staging can be limited to the peritoneal cavity assessment since lymph node involvement is very rare [3]. Both staging and complete gross resection cannot be achieved without an exploration of the pelvic and para-aortic retroperitoneum to exclude disease in nodal tissue. In a retrospective chart review of 68 patients with ovarian GCT, Abu-Rustum et al. [12] reported that clinical stage IA disease was the most common original diagnosis in women who recurred and approximately $15 \%$ of first recurrences appear to involve the retroperitoneum. They concluded that, since retroperitoneal recurrences can occur especially in patients without retroperitoneal staging at initial diagnosis, a standard ovarian surgical staging should be performed in GCT. In our cases, a mean of 32 lymph nodes were removed ( 23 para-aortic) and no complication occurred. The results are even better than those reported by Querleu et al. in their study on the reliability and safety of laparoscopic lymph node dissection in 1,000 gynecologic cancer patients [6]. In our opinion, laparoscopic lymphadenectomy should be performed because of the prognostic role of real stage and the safety of the procedure. In our patients, while bilateral para-aortic lymphadenectomy was performed, only ipsilateral pelvic lymphadenectomy was accomplished. That is why the number of para-aortic lymph-nodes is higher than the pelvic ones.

Generally, patients restaged by laparoscopy benefit of a less invasive and traumatic approach compared with laparotomy and the only disadvantage is the longer operative time. On the contrary, hospital stay is shorter for laparoscopy [8]. In our cases, mean operation time was $195 \mathrm{~min}$. After laparoscopic restaging, one of our patients was discharged on the second and the others on the third postoperative day. In the second case report, the patient was discharged on the first day after second look. In our experience, the advantages of laparoscopy greatly overcome the traditional approach.

In conclusion, laparoscopic restaging of GCT is feasible and allows para-aortic and pelvic lymphadenectomy. Patients restaged by laparoscopy benefit of a less invasive treatment. 


\section{References}

1. Schumer ST, Cannistra SA (2003) Granulosa Cell Tumor of the Ovary. J Clin Oncol 21:1180-1189

2. Stuart GCE, Dawson LM (2003) Update on granulosa cell tumours of the ovary. Curr Opin Obstet Gynecol 15:33-37

3. Leblanc E, Sonoda Y, Narducci F et al (2006) Laparoscopic staging of early ovarian carcinoma. Curr Opin Obstet Gynecol 18:407-412

4. Zhang M, Cheung MK, Shin JY et al (2007) Prognostic factors responsible for survival in sex cord stromal tumors of ovary-An analysis of 376 women. Gynecol Oncol 104:396-400

5. Tozzi R, Schneider A (2005) Laparoscopic treatment of early ovarian cancer. Curr Opin Obstet Gynecol 17:354-358

6. Querleu D, Leblanc E, Cartron G et al (2006) Audit of preoperative and early complications of laparoscopic lymph node dissection in 1000 gynecologic cancer patients. Am J Obstet Gynecol 195:12871292
7. Tozzi R, Kohler C, Ferrara A, Schneider A (2004) Laparoscopic treatment of early ovarian cancer: surgical and survival outcomes. Gynecol Oncol 93:199-203

8. Ghezzi F, Cromi A, Uccella S et al (2007) Laparoscopy versus laparotomy for the surgical management of apparent early stage ovarian cancer. Gynecol Oncol 105:409-413

9. Querleu D (1994) Laparoscopic surgical therapy and staging in a case of early malignant granulosa cell tumor of the ovary. Eur $\mathrm{J}$ Obstet Gynecol Reprod Biol 54:215-217

10. Till H, Schmidt H (2205) Juvenile granulosa cell tumour (JGCT) of the ovary in a 6-year-old girl: laparoscopic resection achieves long-term oncological success. Eur J Pediatr Surg 15:292-294

11. Colombo N, Parma G, Zanagnolo V, Insinga A (2007) Management of ovarian stromal cell tumors. J Clin Oncol 25:2944-29.51

12. Abu-Rustum NR, Restibo A, Ivy J et al (2006) Retroperitoneal nodal metastasis in primary and recurrent granulosa cell tumors of the ovary. Gyn Oncol 103:31-34 